a foundation for connective-tissue formation, which fills up the remainder of the opening. The fear that the flap will cause a damming up of secretion was not justified by Abrashanoff's experience. In a man, aged twenty-seven years, after a stab wound of the chest, there developed an abscess of the lung, which was opened by incision. Ten months after the wound was received a fistula still persisted and communicated with the lung, as shown by bloody expectoration after probing it. The above operation was performed, and in three weeks it was completely cicatrized. It was still closed nine months after the operation. In a woman, aged twenty-three years, during a double salpingectomy for an adhesive pelvic peritonitis, the colon was wounded, and after one and one-half months there still persisted a fistula, 6 cm. deep. After two months it was closed by the same operation. The flap healed in and the escape of feces soon ceased. In an old empyema, operated on twice by Schede's method, there remained a cavity $6 \mathrm{~cm}$. deep, which was filled by such a flap and thus healed.

Concerning the Question of Drainage of the Thorax. - TIEgEL (Zentralbl. f. Chir., 1911, xxxviii, 347) reports the case of a man, aged thirty-seven years, who was admitted to the hospital with a stab wound of the thorax, in an almost pulseless condition. The knife had penetrated the right intercostal space, had divided the second rib and the internal mammary artery, and had produced a deep wound in the upper lobe of the right lung. There was an extensive pneumothorax with a marked cyanosis. Under the administration of oxygen, which improved the dyspnea immediately, the wound was enlarged downward, the wounded vessels ligated, and the wound closed by exact suturing with silk. The skin and muscle wound was closed by sutures down to the lower angle, where an opening was left, three fingers'. breadth below the original wound. Through this oblique canal a strip of iodoform gauze was introduced. On the third day there developed signs of a high grade of pneumothorax, when the tampon was removed and there immediately escaped a quantity of bloody exudate. The breathing, which had been troubled, superficial, and panting, immediately became quiet and deep. In the place of the gauze drain a rubber tube was inserted, which was provided with an improvised valve. Healing followed. As a result of this experience, Tiegel devised a more suitable apparatus for this kind of drainage, which is described in detail. He also provided that the inner end of the tube should lie in the lowest part of the cavity near the spinal column.

\title{
Clinical and Experimental Investigations Concerning the Function of the Stomach after Gastro-enterostomy and Resection of the Pylorus.
} -SchÜller (Mitt. a. d. Grenzegeb. d. Med. u. Chir., 1911, xxii, 715) says that an anterior gastro-enterostomy at the fundus of the stomach with a wide anastomotic opening and an adjacent entero-anastomosis will not cause an alteration in the form, position, or outline on distention of the stomach. Since by the use of the $x$-ray it is seen that during the taking of food, the stomach contents do not pass into the intestine, it is concluded that the anastomotic opening does not interfere with the function of the stomach. The path taken by the 
food after the gastro-enterostomy will depend chiefly upon the movements of the antrum and the permeability of the pylorus. Notwithstanding the gastro-enterostomy, a part of the food always goes through the pylorus, and the closure of the pylorus is the only guarantee of the continued patency of the anastomotic opening. In the normal stomach of a dog, a gastro-enterostomy made in the antral portion of the stomach will provide a more physiological evacuation of the stomach contents, and the opening tends less easily to become closed than when it is made in the fundus portion. It does not prevent, however, the partial passage of the food through the pylorus. For the exclusion of the duodenum, a gastro-enterostomy with closure of the pylorus is indicated. A simple retention from atony of the stomach and inflammatory tumors of the pyloric region, calls for a gastro-enterostomy in the fundus portion of the stomach without closure of the pylorus. In a gastroptosis without retention, a gastroenterostomy is not to be recommended. When, during a gastroenterostomy operation, an unhealed ulcer is found and is not excised, suitable diet and rest of the stomach must be provided. The evacuation of stomach contents through the anastomotic opening will be well toward the normal, but will not exceed the normal. This evacuation does not occur continually, but in jets, and goes mostly toward the efferent loop, rarely through the afferent. The cause of the interruption in the outflow of food is not to be sought in the stomach, but in the intestine. The removal or closure of the pylorus is not associated with as easy an evacuation of the stomach contents as is a simple gastro-enterostomy. The time for evacuation when the pylorus is closed varies. Retention in a resected stomach does not imply an insufficient anastomotic opening. This opening in such an operation should be placed at the deepest part of the stomach. A gastroenterostomy almost always impairs stomach digestion. Without the evidence of lactic acid, a recurrent carcinoma in a resected stomach cannot be diagnosticated. There is no reason after a gastro-enterostomy to reduce the albuminous foods in favor of the fats. Digestive disturbances are to be treated as if no gastro-enterostomy had been done. The stagnation of the escaping stomach contents in the intestinal loop has only a subordinate influence on the development of a jejunal ulcer.

The Results and Prospects of Conservative Treatment of Hourglass Contracture of the Stomach, with a Contribution on the X-ray Diagnosis of the Same.-Schlesrnger (Mitt. a. d. Gren egeb. d. Med. u. Chir., 1911, xxii, 787) says that hourglass contracture of the stomach is generally considered a surgical disease and that it always calls for operation. According to his experience a certain diagnosis and the determination of the degree of the constriction is at times possible only by means of the Röntgen ray. For the differential diagnosis not only is the intermittently spastic contraction of the hourglass stomach observed, but what is more important the formation of folds within the stenosed area and continuously tetanic contraction are maintained by the presence of ulcers, erosions, and stagnation. Occasionally we are able to ameliorate these spasms by internal therapy and sometimes even to cause them to disappear. In this 